ESAIM: COCV 28 (2022) 20

https://doi.org/10.1051/cocv/2022014
ESAIM: Control, Optimisation and Calculus of Variations

www.esaim-cocv.org

\title{
IRREDUCIBILITY OF KURAMOTO-SIVASHINSKY EQUATION DRIVEN BY DEGENERATE NOISE
}

\author{
PENG GaO*
}

\begin{abstract}
In this paper, we study irreducibility of Kuramoto-Sivashinsky equation which is driven by an additive noise acting only on a finite number of Fourier modes. In order to obtain the irreducibility, we first investigate the approximate controllability of Kuramoto-Sivashinsky equation driven by a finite-dimensional force, the proof is based on Agrachev-Sarychev type geometric control approach. Next, we study the continuity of solving operator for deterministic Kuramoto-Sivashinsky equation. Finally, combining the approximate controllability with continuity of solving operator, we establish the irreducibility of Kuramoto-Sivashinsky equation.
\end{abstract}

\section{Mathematics Subject Classification. 60H15.}

Received January 17, 2021. Accepted February 11, 2022.

\section{INTRODUCTION}

The Kuramoto-Sivashinsky equation (KSE)

$$
u_{t}+u_{x x x x}+u_{x x}+u u_{x}=0
$$

was derived independently by Kuramoto et al. in [34-36] as a model for phase turbulence in reaction-diffusion systems and by Sivashinsky in [41] as a model for plane flame propagation, describing the combined influence of diffusion and thermal conduction of the gas on stability of a plane flame front. The KSE is also a mathematical model of reaction-diffusion systems and is related to various pattern formation phenomena where turbulence or chaos appear $[2,21]$. The KSE has been studied as a prototypical example for an infinite-dimensional dynamical system $[16,32,39,42]$.

In order to consider a more realistic model phase turbulence in reaction-diffusion systems, it is sensible to consider some kind of stochastic perturbation represented by a noise term in the equation. Stochastic KSE is an important equation, this model has also attracted more and more attentions, a large amount of work has been devoted to the study of stochastic KSE. References [10, 19] studied the existence and uniqueness of solution for stochastic KSE, [20, 45, 46] discussed the attractor of stochastic KSE, [10, 22] obtained the invariant measure of stochastic KSE, [26, 27] proved the null controllability of stochastic KSE, [9] established the large deviation

Keywords and phrases: Irreducibility, Kuramoto-Sivashinsky equation, degenerate noise, approximate controllability, AgrachevSarychev method.

School of Mathematics and Statistics, and Center for Mathematics and Interdisciplinary Sciences, Northeast Normal University, Changchun 130024, PR China.

* Corresponding author: gaopengjilindaxue@126.com 
principle of stochastic KSE, [31] established the averaging principle for stochastic KSE. We refer readers to the references therein for more interesting results on stochastic KSE.

In this paper, we consider the irreducibility of stochastic KSE driven by highly degenerate noise

$$
\begin{cases}u_{t}+u_{x x x x}+u_{x x}+u u_{x}=b_{1} \dot{\beta}_{1}(t) \sin x+b_{2} \dot{\beta}_{2}(t) \cos x+b_{3} \dot{\beta}_{3}(t) & \text { in } \mathbb{T} \times(0, T), \\ u(0)=u_{0} & \text { in } \mathbb{T},\end{cases}
$$

where $\mathbb{T}=\mathbb{R} / 2 \pi \mathbb{Z}, \beta_{k}, k=1,2,3$ are real valued mutually independent standard Wiener processes defined on a probability space $(\Omega, \mathcal{F}, \mathbb{P})$ and $b_{k}, k=1,2,3$ are real valued numbers. Denote $H:=L^{2}(\mathbb{T}), V:=H^{2}(\mathbb{T})$. According to Theorem 3.4 of [22], we know

If $u_{0} \in H,(1.1)$ admits a unique weak solution $u \in C([0, T] ; H) \mathbb{P}$-a.s. Moreover, $u$ is a Markov process in $H$, which is Feller in $H$.

Let $u\left(t, u_{0}\right)$ denote the unique solution to (1.1) for the initial value $u_{0} \in H$, and we define the transition probabilities $\left\{P\left(t, u_{0}, \cdot\right): t \in[0, T], u_{0} \in H\right\}$ by

$$
P\left(t, u_{0}, \Gamma\right):=\mathbb{P}\left\{u\left(t, u_{0}\right) \in \Gamma\right\}
$$

for all Borel sets $\Gamma$ of $H$ and all $t \in[0, T]$.

Definition 1.1. A family of transition probabilities $\left\{P\left(t, u_{0}, \cdot\right): t \in[0, T], u_{0} \in H\right\}$ is called irreducible in $H$ if for every $u_{0} \in H, t \in[0, T], u_{1} \in H$ and $\varepsilon>0$, we have $P\left(t, u_{0}, B_{H}\left(u_{1}, \varepsilon\right)\right)>0$.

Now, we are in a position to present the main result in this paper.

Theorem 1.2. If $b_{k} \neq 0$ for $k=1,2,3$, then the family $\left\{P\left(t, u_{0}, \cdot\right): t \in[0, T], u_{0} \in H\right\}$ is irreducible in $H$.

The main contribution of this paper is the irreducibility of stochastic KSE driven by the highly degenerate noise. The irreducibility of stochastic partial differential equations (SPDEs) has attracted many authors' attention, see $[6-8,18,22,23,43,44]$ and references within. However, to the best knowledge of the author, the irreducibility of SPDEs is generally established for non-degenerate noise, the result for the case of degenerate noise is far less studied. The irreducibility is a fundamental concept in stochastic dynamic system, and it plays a crucial role in the research of ergodic theory, moderate deviation principle. More precisely, the main reason for the study of the irreducibility property is its relevance in ergodic theory, and in the analysis of the uniqueness and ergodicity of invariant measures. The property of irreducibility is at the core of the problem, it is sufficient to obtain the ergodic result by adding the strong Feller property, see the classical work [17, 24]. Another application of the irreducibility is to establish moderate deviation principle of SPDEs, in general, the moderate deviation principle is obtained by verifying the irreducibility and some Lyapunov condition for SPDEs, see [43].

The proof of Theorem 1.2 relies on the approximate controllability of KSE (see Thm. 3.3) and the continuity of the mapping noise $\mapsto$ solution along the controllers (see Prop. 4.2). It is well known that one usually solves a control problem to prove the irreducibility for SPDEs. However, due to the highly degenerate of the noise of (1.1), the control problem of the associated deterministic system is much harder than those in the usual case, the traditional methods and techniques in the existing literature are no longer feasible. The novelty of this paper is that we introduce the Agrachev-Sarychev type geometric control approach to overcome this difficulty. More precisely, with the help of asymptotic property of operator $\mathcal{R}$ and saturating property of subspace of $\mathcal{H}$, we can prove the approximate controllability of the associated deterministic system with finite-dimensional control.

The control theory is known to be a useful tool in the study of stochastic systems with non-degenerate noise, see [17] and references therein for more interesting results on this topic. In recent years, stochastic system with highly degenerate noise is attracting more and more people's attention, this paper shows that the AgrachevSarychev approach is a powerful tool for the study of stochastic systems with highly degenerate noise. Let us mention that the method and framework in this paper are quite general and can be adapted to degenerate problems for more SPDEs. 
The rest of this paper is organized as follows: In Section 2, we give some preliminary results which will be used in this paper. Section 3 is devoted to the approximate controllability of KSE driven by a finite-dimensional force. The proof of Theorem 1.2 is given in Section 4.

\section{PreliminaRies}

If $u \in L^{2}(\mathbb{T})$, it can be written as $u(x)=\sum_{k \in \mathbb{Z}} u_{k} e^{i k x}$ with $u_{k} \in \mathbb{C}$ and $u_{-k}=\overline{u_{k}}$, the norm in $H^{s}(\mathbb{T})$ is defined by $\|\cdot\|_{s}$ :

$$
\|u\|_{s}^{2}:=\sum_{k \in \mathbb{Z}}\left(1+|k|^{2}\right)^{s}\left|u_{k}\right|^{2}
$$

For $s=0,\|\cdot\|_{0}:=\|\cdot\|$.

For any $s \geq 0$ and $u=\sum_{k \in \mathbb{Z}} u_{k} e^{i k x} \in H^{s}(\mathbb{T})$, we define

$$
\partial_{x}^{s} u:=\sum_{k \in \mathbb{Z}}|k|^{s} u_{k} e^{i k x}
$$

In this paper, we will use the following inequalities.

Lemma 2.1. If $a, b \in \mathbb{R}, p>0$, it holds that

$$
(|a|+|b|)^{p} \leq\left\{\begin{array}{lc}
|a|^{p}+|b|^{p} & 0<p \leq 1, \\
2^{p-1}\left(|a|^{p}+|b|^{p}\right) & p>1 .
\end{array}\right.
$$

Lemma 2.2. (Gronwall inequality) Let $y(t)$ be a nonnegative function and $a \in \mathbb{R}, f \in L_{\text {loc }}^{1}(\mathbb{R})$, if $y^{\prime} \leq-a y+f$, we have

$$
y(t) \leq y(s) e^{-a(t-s)}+\int_{s}^{t} e^{-a(t-\tau)} f(\tau) \mathrm{d} \tau .
$$

Lemma 2.3. (Young inequality) Let $a, b \in[0,+\infty)$ and $\varepsilon>0$, then we have

$$
a b \leq \varepsilon^{-p} \frac{a^{p}}{p}+\varepsilon^{q} \frac{b^{q}}{q}
$$

where $1<p<\infty, \frac{1}{p}+\frac{1}{q}=1$.

Lemma 2.4. (See [1]) Let $0 \leq s_{1}<s_{2}$, for any $\varepsilon>0$ and $u \in H^{s_{2}}(\mathbb{T})$, there exists a constant $C(\varepsilon)>0$ such that

$$
\|u\|_{s_{1}} \leq \varepsilon\left\|\partial_{x}^{s_{2}} u\right\|+C(\varepsilon)\|u\| .
$$

Lemma 2.5. (See [1]) Let $s>\frac{1}{2}$, then for any $u \in H^{s}(\mathbb{T})$, we have

$$
\|u\|_{L^{\infty}(\mathbb{T})} \leq C\|u\|_{s}
$$




\section{Approximate controllability of KSE}

We first consider the approximate controllability of the following KSE driven by a finite-dimensional force:

$$
\begin{cases}u_{t}+u_{x x x x}+u_{x x}+u u_{x}=h(x, t)+\eta(x, t) & \text { in } \mathbb{T} \times(0, T), \\ u(0)=u_{0} & \text { in } \mathbb{T}\end{cases}
$$

where the function $\eta$ plays the role of the control.

Definition 3.1. For $s \geq 0$, we shall say that (3.1) is approximately controllable by $\mathcal{H}$-valued control if for any initial point $u_{0} \in H^{s}(\mathbb{T})$, any target $u_{1} \in H^{s}(\mathbb{T})$, and any number $\varepsilon>0$, there is a control $\eta \in L^{2}(0, T ; \mathcal{H})$ and a unique solution $u$ of problem (3.1) defined on the interval $[0, T]$ such that

$$
\left\|u(T)-u_{1}\right\|_{s} \leq \varepsilon
$$

Let $\mathcal{H}$ be a finite-dimensional subspace of $H^{s+4}(\mathbb{T})$. We define a non-decreasing sequence of finite-dimensional subspaces:

$$
\mathcal{H}_{0}:=\mathcal{H}, \quad \mathcal{H}_{j}:=\mathcal{F}\left(\mathcal{H}_{j-1}\right), j \geq 1, \quad \mathcal{H}_{\infty}:=\bigcup_{j=1}^{\infty} \mathcal{H}_{j}
$$

where

$$
\mathcal{F}(\mathcal{H}):=\operatorname{span}\left\{\eta-\sum_{i=1}^{N} u_{i} \partial_{x} u_{i}: \eta, u_{i} \in \mathcal{H}, i=1,2, \cdots, N\right\}
$$

Definition 3.2. We say that $\mathcal{H}$ is saturating if $\mathcal{H}_{\infty}$ is dense in $H^{s}(\mathbb{T})$.

Now, we are in a position to present the main result in this section.

Theorem 3.3. Let $T>0, s>\frac{1}{2}, u_{0} \in H^{s}(\mathbb{T})$ and $h \in L^{2}\left(0, T ; H^{s-2}(\mathbb{T})\right)$. If $\mathcal{H}$ is saturating, then (3.1) is approximately controllable by $\mathcal{H}$-valued control.

The proof of Theorem 3.3 is based on the idea from the works of Agrachev and Sarychev [3-5], who studied the approximate controllability of the 2D Navier-Stokes and Euler systems by finite-dimensional forces. The Agrachev-Sarychev method is also applied to other equations, see $[11,40]$ and the literatures therein. The controllability of KSE has attracted many authors' attention, see [12-15, 25-30].

Here, we give examples of saturating spaces. $\mathcal{I}$ is called a generator if $\mathcal{I} \subset \mathbb{Z}$ is a finite symmetric set containing 0 and any integer is a linear combination of elements of $\mathcal{I}$ with integer coefficients. We define the space

$$
\mathcal{H}(\mathcal{I})=\operatorname{span}\{\sin (m x), \cos (m x): m \in \mathcal{I}\}
$$

Proposition 3.4. If $\mathcal{I}$ is a generator, then the space $\mathcal{H}(\mathcal{I})$ is saturating. In particular, $\mathcal{E}:=\operatorname{span}\{\sin x, \cos x, 1\}$ is saturating.

The proof of Proposition 3.4 is done later in Section 3.4. 


\subsection{Well-posedness of generalised KSE}

We consider the following generalised KSE:

$$
\begin{cases}u_{t}+(u+\zeta)_{x x x x}+(u+\zeta)_{x x}+(u+\zeta)(u+\zeta)_{x}=\varphi & \text { in } \mathbb{T} \times(0, T), \\ u(0)=u_{0} & \text { in } \mathbb{T} .\end{cases}
$$

For any $T>0$ and $s \geq 0$, we define the space

$$
\mathcal{X}_{T, s}:=C\left([0, T] ; H^{s}(\mathbb{T})\right) \cap L^{2}\left(0, T ; H^{s+2}(\mathbb{T})\right)
$$

endowed with the norm

$$
\|u\|_{\mathcal{X}_{T, s}}:=\|u\|_{C\left([0, T] ; H^{s}(\mathbb{T})\right)}+\|u\|_{L^{2}\left(0, T ; H^{s+2}(\mathbb{T})\right)} .
$$

Define the operator $P=-\partial_{x}^{4}$ with domain $\mathcal{D}(P)=H^{4}(\mathbb{T})$, we know that $P$ generates a semigroup $\{S(t)\}_{\{t \geq 0\}}$ in $L^{2}(\mathbb{T})$. Moreover, we have the following important property:

Lemma 3.5. For any $T>0$ and $s \geq 0$, the semigroup satisfies that

$$
\begin{aligned}
& \left\|S(\cdot) y_{0}\right\|_{\mathcal{X}_{T, s}} \leq C\left\|y_{0}\right\|_{s}, \quad \forall y_{0} \in H^{s}(\mathbb{T}), \\
& \left\|\int_{0}^{\cdot} S(\cdot-\tau) f(\tau) \mathrm{d} \tau\right\|_{\mathcal{X}_{T, s}} \leq C\|f\|_{L^{2}\left(0, T ; H^{s-2}(\mathbb{T})\right)}, \quad \forall f \in L^{2}\left(0, T ; H^{s-2}(\mathbb{T})\right),
\end{aligned}
$$

where $C=C(T, s)$ is a positive constant that increases in the first variable.

The proof of Lemma 3.5 is done later in Section 3.5.

Proposition 3.6. For any $T>0$ and $s>\frac{1}{2}$, let $u_{0} \in H^{s}(\mathbb{T}), \zeta \in L^{4}\left(0, T ; H^{s+2}(\mathbb{T})\right)$ and $\varphi \in L^{2}\left(0, T ; H^{s-2}(\mathbb{T})\right)$. Then system (3.2) admits a unique solution $u \in \mathcal{X}_{T, s}$. Moreover, let $\mathcal{R}$ be the mapping taking a triple $\left(u_{0}, \zeta, \varphi\right)$ to the solution of $(3.2)$, for $\hat{u}_{0} \in H^{s}(\mathbb{T})$ and $\hat{\varphi} \in L^{2}\left(0, T ; H^{s-2}(\mathbb{T})\right)$, we have

$$
\left\|\mathcal{R}\left(u_{0}, 0, \varphi\right)-\mathcal{R}\left(\hat{u}_{0}, 0, \hat{\varphi}\right)\right\|_{\mathcal{X}_{T, s}} \leq C\left(\left\|u_{0}-\hat{u}_{0}\right\|_{s}+\|\varphi-\hat{\varphi}\|_{L^{2}\left(0, T ; H^{s-2}(\mathbb{T})\right)}\right) .
$$

Proof of Proposition 3.6. Before we prove Proposition 3.6, we introduce the following important mathematical setting: Throughout the paper, the letter $C$ denotes unessential positive constant whose value may change in different occasions, which may vary from line to line. We will write the dependence of constant on parameters explicitly if it is essential.

First, we consider the existence and uniqueness of the solution for (3.2).

Let $t \in[0, \theta] \subset[0, T]$ and $v \in \mathcal{X}_{\theta, s}$, we set

$$
\Phi(v)(t)=S(t) u_{0}+\int_{0}^{t} S(t-\tau) F(v)(\tau) \mathrm{d} \tau,
$$

where

$$
F(v)=-\partial_{x}^{2} v-\partial_{x}^{4} \zeta-\partial_{x}^{2} \zeta-(v+\zeta) \partial_{x}(v+\zeta)+\varphi .
$$

Since $H^{s}(\mathbb{T})\left(s>\frac{1}{2}\right)$ is a Banach algebra, we get

$$
\left\|(v+\zeta) \partial_{x}(v+\zeta)\right\|_{s-2} \leq C\left\|(v+\zeta)^{2}\right\|_{s-1} \leq C\left\|(v+\zeta)^{2}\right\|_{s} \leq C\|v+\zeta\|_{s}^{2} \leq C\left(\|v\|_{s}^{2}+\|\zeta\|_{s}^{2}\right),
$$


this implies that

$$
\|F(v)\|_{s-2} \leq C\left(\|v\|_{s}+\|\zeta\|_{s+2}+\|v\|_{s}^{2}+\|\zeta\|_{s}^{2}+\|\varphi\|_{s-2}\right) .
$$

According to this estimate, we have

$$
\begin{aligned}
\|F(v)\|_{L^{2}\left(0, \theta ; H^{s-2}(\mathbb{T})\right) \leq} & C \theta^{\frac{1}{2}}\left(\|v\|_{\mathcal{X}_{\theta, s}}+\|v\|_{\mathcal{X}_{\theta, s}}^{2}\right) \\
& +C\left(\|\zeta\|_{L^{2}\left(0, T ; H^{s+2}(\mathbb{T})\right)}+\|\zeta\|_{L^{4}\left(0, T ; H^{s}(\mathbb{T})\right)}^{2}+\|\varphi\|_{L^{2}\left(0, T ; H^{s-2}(\mathbb{T})\right)}\right) \\
\leq & C \theta^{\frac{1}{2}}\left(\|v\|_{\mathcal{X}_{\theta, s}}+\|v\|_{\mathcal{X}_{\theta, s}}^{2}\right) \\
& +C\left(\|\zeta\|_{L^{4}\left(0, T ; H^{s+2}(\mathbb{T})\right)}+\|\zeta\|_{L^{4}\left(0, T ; H^{s+2}(\mathbb{T})\right)}^{2}+\|\varphi\|_{L^{2}\left(0, T ; H^{s-2}(\mathbb{T})\right)}\right) .
\end{aligned}
$$

It follows from (3.3) that

$$
\begin{aligned}
\|\Phi(v)\|_{\mathcal{X}_{\theta, s} \leq} \leq & C\left(\left\|u_{0}\right\|_{s}+\|F(v)\|_{L^{2}\left(0, \theta ; H^{s-2}(\mathbb{T})\right)}\right) \\
\leq & C_{1}\left(\left\|u_{0}\right\|_{s}+\|\zeta\|_{L^{4}\left(0, T ; H^{s+2}(\mathbb{T})\right)}+\|\zeta\|_{L^{4}\left(0, T ; H^{s+2}(\mathbb{T})\right)}^{2}+\|\varphi\|_{L^{2}\left(0, T ; H^{s-2}(\mathbb{T})\right)}\right) \\
& +C_{2} \theta^{\frac{1}{2}}\left(\|v\|_{\mathcal{X}_{\theta, s}}+\|v\|_{\mathcal{X}_{\theta, s}}^{2}\right),
\end{aligned}
$$

where $C_{1}, C_{2}$ depend on $T$ but independent of $\theta$.

For any $v_{1}, v_{2} \in \mathcal{X}_{\theta, s}$, the same argument shows that

$$
\begin{aligned}
\left\|\Phi\left(v_{1}\right)-\Phi\left(v_{2}\right)\right\|_{\mathcal{X}_{\theta, s}} & \leq C\left\|F\left(v_{1}\right)-F\left(v_{2}\right)\right\|_{L^{2}\left(0, \theta ; H^{s-2}(\mathbb{T})\right)} \\
& \leq C_{3}\left[\theta^{\frac{1}{2}}\left(1+\left\|v_{1}\right\|_{\mathcal{X}_{\theta, s}}+\left\|v_{2}\right\|_{\mathcal{X}_{\theta, s}}\right)+\theta^{\frac{1}{4}}\|\zeta\|_{L^{4}\left(0, T ; H^{s+2}(\mathbb{T})\right)}\right]\left\|v_{1}-v_{2}\right\|_{\mathcal{X}_{\theta, s}} .
\end{aligned}
$$

Set

$$
R:=2 C_{1}\left(\left\|u_{0}\right\|_{s}+\|\zeta\|_{L^{4}\left(0, T ; H^{s+2}(\mathbb{T})\right)}+\|\zeta\|_{L^{4}\left(0, T ; H^{s+2}(\mathbb{T})\right)}^{2}+\|\varphi\|_{L^{2}\left(0, T ; H^{s-2}(\mathbb{T})\right)}\right) .
$$

If $v, v_{1}, v_{2} \in B_{\mathcal{X}_{\theta, s}}(R):=\left\{u \in \mathcal{X}_{\theta, s} \mid\|u\|_{\mathcal{X}_{\theta, s}} \leq R\right\}$, we can choose $\theta$ sufficiently small such that

$$
\begin{aligned}
& C_{2} \theta^{\frac{1}{2}}(1+R) \leq \frac{1}{2}, \\
& C_{3}\left[\theta^{\frac{1}{2}}(1+2 R)+\theta^{\frac{1}{4}}\|\zeta\|_{L^{4}\left(0, T ; H^{s+2}(\mathbb{T})\right)}\right] \leq \frac{1}{2},
\end{aligned}
$$

then according to (3.5) and (3.6), we have

$$
\begin{aligned}
& \|\Phi(v)\|_{\mathcal{X}_{\theta, s}} \leq \frac{R}{2}+C_{2} \theta^{\frac{1}{2}}(1+R)\|v\|_{\mathcal{X}_{\theta, s}} \leq R, \\
& \left\|\Phi\left(v_{1}\right)-\Phi\left(v_{2}\right)\right\|_{\mathcal{X}_{\theta, s}} \leq \frac{1}{2}\left\|v_{1}-v_{2}\right\|_{\mathcal{X}_{\theta, s}} .
\end{aligned}
$$

This implies that $\Phi$ has a unique fixed point $u \in B_{\mathcal{X}_{\theta, s}}(R)$ which will be the solution of (3.2) for small $\theta$.

To obtain the global existence, we will prove the following a prior estimate

$$
\|u\|_{\mathcal{X}_{T, s}} \leq C
$$

where $C=C\left(T,\left\|u_{0}\right\|_{s},\|\zeta\|_{L^{4}\left(0, T ; H^{s+2}(\mathbb{T})\right)},\|\varphi\|_{L^{2}\left(0, T ; H^{s-2}(\mathbb{T})\right)}\right)$. 
For $s=0$, taking the scalar product in $L^{2}(\mathbb{T})$ of equation (3.2) with $u$, noting the facts that

$$
\begin{aligned}
& \int_{\mathbb{T}}(\zeta u)_{x} u \mathrm{~d} x=-\int_{\mathbb{T}} \zeta u u_{x} \mathrm{~d} x=\frac{1}{2} \int_{\mathbb{T}} \zeta_{x} u^{2} \mathrm{~d} x \leq C\|\zeta\|_{2}\|u\|^{2}, \\
& \int_{\mathbb{T}} \zeta \zeta_{x} u \mathrm{~d} x \leq\left\|\zeta \zeta_{x}\right\|\|u\| \leq C\|\zeta\|_{2}^{2}\|u\|,
\end{aligned}
$$

we have

$$
\begin{aligned}
\frac{1}{2} \frac{\mathrm{d}}{\mathrm{d} t}\|u\|^{2}+\left\|\partial_{x}^{2} u\right\|^{2} & \leq C\left(\left\|\partial_{x}^{2} u\right\|\|u\|+\|\zeta\|_{2}\left\|\partial_{x}^{2} u\right\|+\|\zeta\|_{2}\|u\|^{2}+\|\zeta\|_{2}^{2}\|u\|+\|\varphi\|_{-2}\|u\|_{2}\right) \\
& \leq \frac{1}{2}\left\|\partial_{x}^{2} u\right\|^{2}+C\left(1+\|\zeta\|_{2}\right)\|u\|^{2}+C\left(1+\|\zeta\|_{2}^{4}+\|\varphi\|_{-2}^{2}\right) .
\end{aligned}
$$

By Gronwall's inequality, (3.7) holds for $s=0$.

For $s>0$, we can prove (3.7) by induction. More precisely, let $k$ be any nonnegative integer, assume that if we have (3.7) for $s=k$, we claim that (3.7) holds for $k<s \leq k+1$.

To this purpose, taking the scalar product in $L^{2}(\mathbb{T})$ of equation $(3.2)$ with $\partial_{x}^{2 s} u$, we can obtain that

$$
\begin{aligned}
& \frac{1}{2} \frac{\mathrm{d}}{\mathrm{d} t}\left\|\partial_{x}^{s} u\right\|^{2}+\left\|\partial_{x}^{s+2} u\right\|^{2} \\
\leq & C\left(\left\|\partial_{x}^{s+2} u\right\|\left\|\partial_{x}^{s} u\right\|+\|\zeta\|_{s+2}\left\|\partial_{x}^{s+2} u\right\|-\left(u u_{x}+(u \zeta)_{x}, \partial_{x}^{2 s} u\right)+\|\zeta\|_{s+2}^{2}\left\|\partial_{x}^{s} u\right\|+\|\varphi\|_{s-2}\|u\|_{s+2}\right) \\
\leq & \frac{1}{4}\left\|\partial_{x}^{s+2} u\right\|^{2}+C\left\|\partial_{x}^{s} u\right\|^{2}+C\left(1+\|u\|^{2}+\|\zeta\|_{s+2}^{4}+\|\varphi\|_{s-2}^{2}\right)-\left(u u_{x}+(u \zeta)_{x}, \partial_{x}^{2 s} u\right) .
\end{aligned}
$$

Our task is reduced to estimate $\left(u u_{x}+(u \zeta)_{x}, \partial_{x}^{2 s} u\right)$. Applying Young's inequality in Lemma 2.3 and interpolation inequality in Lemma 2.4, we have

$$
\begin{aligned}
\left|\left((u \zeta)_{x}, \partial_{x}^{2 s} u\right)\right| & \leq\left|\left(\partial_{x}^{s+1}(u \zeta), \partial_{x}^{s} u\right)\right| \\
& \leq\|u \zeta\|_{s+1}\left\|\partial_{x}^{s} u\right\| \\
& \leq C\|u\|_{s+1}\|\zeta\|_{s+1}\left\|\partial_{x}^{s} u\right\| \\
& \leq C\left(\left\|\partial_{x}^{s+2} u\right\|+\|u\|\right)\|\zeta\|_{s+1}\left\|\partial_{x}^{s} u\right\| \\
& \leq \frac{1}{8}\left\|\partial_{x}^{s+2} u\right\|^{2}+C\left(1+\|\zeta\|_{s+1}^{2}\right)\left\|\partial_{x}^{s} u\right\|^{2}+C\|\zeta\|_{s+1}^{2}\|u\|^{2} .
\end{aligned}
$$

We will prove that for any $k<s \leq k+1, k$ is any nonnegative integer, it holds that

$$
\left|\left(u u_{x}, \partial_{x}^{2 s} u\right)\right| \leq \frac{1}{8}\left\|\partial_{x}^{s+2} u\right\|^{2}+C
$$

Indeed, if $k=0,0<s \leq 1$, then $2 s+1 \leq s+2$, then for any $\varepsilon>0$, it holds that

$$
\begin{aligned}
\left|\left(u u_{x}, \partial_{x}^{2 s} u\right)\right| & \leq C\|u\|_{L^{\infty}(\mathbb{T})}\|u\|\left\|\partial_{x}^{2 s+1} u\right\| \\
& \leq C\|u\|_{1}\|u\|\|u\|_{s+2} \\
& \leq C\|u\|\left(\varepsilon\left\|\partial_{x}^{s+2} u\right\|^{2}+C(\varepsilon)\|u\|^{2}\right)
\end{aligned}
$$

where we have applied the interpolation inequality in Lemma 2.4 to obtain $\|u\|_{1}\|u\|_{s+2} \leq \varepsilon\left\|\partial_{x}^{s+2} u\right\|^{2}+C(\varepsilon)\|u\|^{2}$. According to (3.7) for $s=0$, we can choose $\varepsilon$ small enough such that (3.10) holds. 
If $k \geq 1, k<s \leq k+1$, we have $2 s-k+1 \leq s+2$, then it follows that

$$
\begin{aligned}
\left|\left(u u_{x}, \partial_{x}^{2 s} u\right)\right| & \leq C\left|\left(\partial_{x}^{k}\left(u^{2}\right), \partial_{x}^{2 s-k+1} u\right)\right| \\
& \leq C\|u\|_{k}^{2}\|u\|_{s+2} \\
& \leq C\|u\|_{k}^{4}+\frac{1}{8}\|u\|_{s+2}^{2} \\
& \leq \frac{1}{8}\left\|\partial_{x}^{s+2} u\right\|^{2}+C\left(\|u\|_{k}^{4}+\|u\|^{2}\right) .
\end{aligned}
$$

Due to the assumption that (3.7) holds for $s=k$, the above estimate implies that (3.10) holds.

Combining estimates (3.8)-(3.10), we conclude that

$$
\frac{\mathrm{d}}{\mathrm{d} t}\left\|\partial_{x}^{s} u\right\|^{2} \leq C\left(1+\|\zeta\|_{s+2}^{4}\right)\left\|\partial_{x}^{s} u\right\|^{2}+C\left(1+\|\zeta\|_{s+2}^{4}+\|\varphi\|_{s-2}^{2}\right) .
$$

Applying Gronwall's inequality again, we can obtain (3.7) for $k<s \leq k+1$. This implies that (3.7) holds for any $s \geq 0$. Namely, we get the existence of the solution on $[0, T]$.

For the uniqueness, let $u_{1}, u_{2} \in \mathcal{X}_{T, s}$ be two solutions of (3.2), then $v=u_{1}-u_{2}$ satisfies

$$
\begin{cases}v_{t}+v_{x x x x}+v_{x x}+u_{1} v_{x}+v u_{2 x}+(\zeta v)_{x}=0 & \text { in } \mathbb{T} \times(0, T), \\ v(0)=0 & \text { in } \mathbb{T} .\end{cases}
$$

Repeating the above arguments, we get that

$$
\frac{\mathrm{d}}{\mathrm{d} t}\|v\|^{2} \leq C\left(1+\|\zeta\|_{2}+\left\|u_{1}\right\|_{2}+\left\|u_{2}\right\|_{2}\right)\|v\|^{2},
$$

which implies that $v \equiv 0$ in view of the Gronwall's inequality.

Finally, we prove (3.4). To this purpose, let $\hat{u}$ be the solution of $(3.2)$ with $\left(\hat{u}_{0}, 0, \hat{\varphi}\right)$ and $w=u-\hat{u}$, then $w$ is a solution of problem

$$
\begin{cases}w_{t}+w_{x x x x}+w_{x x}+u w_{x}+w \hat{u}_{x}=\eta & \text { in } \mathbb{T} \times(0, T), \\ w(0)=w_{0} & \text { in } \mathbb{T},\end{cases}
$$

where $w_{0}=u_{0}-\hat{u}_{0}$ and $\eta=\varphi-\hat{\varphi}$. Applying similar methods as above, we can obtain that

$$
\begin{aligned}
& \frac{\mathrm{d}}{\mathrm{d} t}\|w\|^{2}+\left\|\partial_{x}^{2} w\right\|^{2} \leq C\left(1+\|u\|_{2}+\|\hat{u}\|_{2}\right)\|w\|^{2}+C\|\eta\|_{-2}^{2}, \\
& \frac{\mathrm{d}}{\mathrm{d} t}\left\|\partial_{x}^{s} w\right\|^{2}+\left\|\partial_{x}^{s+2} w\right\|^{2} \leq C\left(1+\|u\|_{s+2}^{2}+\|\hat{u}\|_{s+2}^{2}\right)\left\|\partial_{x}^{s} w\right\|^{2}+C\left(\|\eta\|_{s-2}^{2}+\|w\|^{2}\right), \quad s>0 .
\end{aligned}
$$

Thus, (3.4) follows from Gronwall's inequality.

This ends the proof of Proposition 3.6.

\subsection{Asymptotic property}

The following asymptotic property plays an important role in the proof of approximate controllability. 
Proposition 3.7. Let $T>0$ and $s>\frac{1}{2}$. For any $u_{0}, \eta \in H^{s+2}(\mathbb{T}), \zeta \in H^{s+4}(\mathbb{T}), h \in L^{2}\left(0, T ; H^{s-2}(\mathbb{T})\right)$, there is a number $\delta_{0} \in(0,1)$ such that for any $\delta \in\left(0, \delta_{0}\right)$, the following limit holds

$$
\mathcal{R}_{\delta}\left(u_{0}, \delta^{-\frac{1}{2}} \zeta, h+\delta^{-1} \eta\right) \longrightarrow u_{0}+\eta-\zeta \zeta_{x} \quad \text { in } H^{s}(\mathbb{T}) \quad \text { as } \delta \rightarrow 0 .
$$

Proof. Let us take any $\delta>0$ and consider the equation

$$
u_{t}+\left(u+\delta^{-\frac{1}{2}} \zeta\right)_{x x x x}+\left(u+\delta^{-\frac{1}{2}} \zeta\right)_{x x}+\left(u+\delta^{-\frac{1}{2}} \zeta\right)\left(u+\delta^{-\frac{1}{2}} \zeta\right)_{x}=h+\delta^{-1} \eta .
$$

By Proposition 3.6, (3.12) has a unique solution in $\mathcal{X}_{T, s}$. Make a time substitution and consider the functions

$$
\begin{aligned}
& w(t):=u_{0}+t\left(\eta-\zeta \zeta_{x}\right), \\
& v(t):=u(\delta t)-w(t),
\end{aligned}
$$

where $t \leq 1 \wedge \delta^{-1} T$. Then $v$ is a solution of the following system

$$
\left\{\begin{array}{l}
v_{t}+\delta\left(v+w+\delta^{-\frac{1}{2}} \zeta\right)_{x x x x}+\delta\left(v+w+\delta^{-\frac{1}{2}} \zeta\right)_{x x} \\
\quad+\delta\left(v+w+\delta^{-\frac{1}{2}} \zeta\right)\left(v+w+\delta^{-\frac{1}{2}} \zeta\right)_{x}-\zeta \zeta_{x}=\delta h \\
v(0)=0
\end{array}\right.
$$

Taking the scalar product in $L^{2}(\mathbb{T})$ of equation (3.13) with $v$, we can obtain that

$$
\begin{aligned}
\frac{1}{2} \frac{\mathrm{d}}{\mathrm{d} t}\|v\|^{2}+\delta\left\|\partial_{x}^{2} v\right\|^{2} \leq & \delta\|v\|\left\|\partial_{x}^{2} v\right\|+\delta\|w\|_{2}\left\|\partial_{x}^{2} v\right\|+\delta^{\frac{1}{2}}\|\zeta\|_{4}\|v\|+C \delta\|w\|_{1}^{2}\|v\|+C \delta\|w\|_{2}\|v\|^{2} \\
& +C \delta^{\frac{1}{2}}\|\zeta\|_{2}\|v\|^{2}+C \delta^{\frac{1}{2}}\|w\|_{1}\|\zeta\|_{1}\|v\|+C \delta\|h\|_{-2}\left(\|v\|+\left\|\partial_{x}^{2} v\right\|\right) \\
\leq & \frac{\delta}{2}\left\|\partial_{x}^{2} v\right\|^{2}+C \delta^{\frac{1}{2}}\left(1+\|h\|_{-2}^{2}+\|v\|^{2}\right)
\end{aligned}
$$

this implies that

$$
\frac{\mathrm{d}}{\mathrm{d} t}\|v\|^{2} \leq C \delta^{\frac{1}{2}}\left(1+\|h\|_{-2}^{2}+\|v\|^{2}\right) .
$$

Applying Gronwall's inequality, we have

$$
\|v(t)\|^{2} \leq C \delta^{\frac{1}{2}} \quad \text { for } 0<t \leq 1 \wedge\left(\delta^{-1} T\right) .
$$

Then taking the scalar product in $L^{2}(\mathbb{T})$ of equation (3.13) with $\partial_{x}^{2 s} v$, it follows that

$$
\begin{aligned}
& \frac{1}{2} \frac{\mathrm{d}}{\mathrm{d} t}\left\|\partial_{x}^{s} v\right\|^{2}+\delta\left\|\partial_{x}^{s+2} v\right\|^{2} \\
\leq & \delta\left\|\partial_{x}^{s} v\right\|\left\|\partial_{x}^{s+2} v\right\|+\delta\|w\|_{s+2}\left\|\partial_{x}^{s+2} v\right\|+\delta^{\frac{1}{2}}\|\zeta\|_{s+4}\left\|\partial_{x}^{s} v\right\|+N(w, \zeta, v)+\delta\|h\|_{s-2}\left(\left\|\partial_{x}^{s+2} v\right\|+\|v\|\right) \\
\leq & \frac{\delta}{4}\left\|\partial_{x}^{s+2} v\right\|^{2}+C \delta^{\frac{1}{2}}\left(1+\|h\|_{s-2}^{2}+\left\|\partial_{x}^{s} v\right\|^{2}\right)+N(w, \zeta, v),
\end{aligned}
$$

where

$$
N(w, \zeta, v)=\left(\delta v v_{x}+\delta w w_{x}+\delta(w v)_{x}+\delta^{\frac{1}{2}}(\zeta v)_{x}+\delta^{\frac{1}{2}}(\zeta w)_{x}, \partial_{x}^{2 s} v\right)
$$


The first term in $N(w, \zeta, v)$ can be estimated as follows:

Since $H^{s}(\mathbb{T})\left(s>\frac{1}{2}\right)$ is an algebra, it follows from the interpolation inequality in Lemma 2.4 that

$$
\begin{aligned}
\left|\left(\delta v v_{x}, \partial_{x}^{2 s} v\right)\right| & =\frac{\delta}{2}\left|\left(\partial_{x}^{s}\left(v^{2}\right), \partial_{x}^{s+1} v\right)\right| \\
& \leq C \delta\left\|v^{2}\right\|\left\|_{s}\right\| \partial_{x}^{s+1} v \| \\
& \leq C \delta\|v\|_{s}^{2}\left\|\partial_{x}^{s+1} v\right\| \\
& \leq C \delta\left(\left\|\partial_{x}^{s} v\right\|^{2}+\|v\|^{2}\right)\left(\left\|\partial_{x}^{s+2} v\right\|+\|v\|\right) \\
& \leq \frac{\delta}{8}\left\|\partial_{x}^{s+2} v\right\|^{2}+C \delta\left(\left\|\partial_{x}^{s} v\right\|^{4}+\|v\|^{4}+1\right) .
\end{aligned}
$$

Therefore, for any $s>\frac{1}{2}$,

$$
\left|\left(\delta v v_{x}, \partial_{x}^{2 s} v\right)\right| \leq \frac{\delta}{8}\left\|\partial_{x}^{s+2} v\right\|^{2}+C \delta\left(\left\|\partial_{x}^{s} v\right\|^{4}+1\right)
$$

By the similar method as above, we can deduce that

$$
\left|\left(\delta(w v)_{x}, \partial_{x}^{2 s} v\right)\right| \leq \frac{\delta}{8}\left\|\partial_{x}^{s+2} v\right\|^{2}+C \delta\left(\left\|\partial_{x}^{s} v\right\|^{2}+1\right) .
$$

Next, we consider $\left(\delta^{\frac{1}{2}}(\zeta v)_{x}, \partial_{x}^{2 s} v\right)$. Since $s>\frac{1}{2}$, with the help of the interpolation inequality in Lemma 2.4, we have

$$
\begin{aligned}
\left|\left(\delta^{\frac{1}{2}}(\zeta v)_{x}, \partial_{x}^{2 s} v\right)\right| & =\left|\delta^{\frac{1}{2}}\left(\partial_{x}^{s}(\zeta v), \partial_{x}^{s+1} v\right)\right| \\
& \leq C \delta^{\frac{1}{2}}\|\zeta\|_{s}\|v\|_{s}\left\|\partial_{x}^{s+1} v\right\| \\
& \leq C \delta^{\frac{1}{2}}\left(\left\|\partial_{x}^{s} v\right\|+\|v\|\right)\left(\delta^{\frac{1}{4}}\left\|\partial_{x}^{s+2} v\right\|+\delta^{-\frac{1}{4}}\left\|\partial_{x}^{s} v\right\|\right) \\
& \leq \frac{\delta}{8}\left\|\partial_{x}^{s+2} v\right\|^{2}+C \delta^{\frac{1}{4}}\left(\left\|\partial_{x}^{s} v\right\|^{2}+1\right)
\end{aligned}
$$

Then, consideration is given to the remaining terms in $N(w, \zeta, v)$,

$$
\begin{aligned}
\left|\left(\delta w w_{x}, \partial_{x}^{2 s} v\right)\right|+\left|\left(\delta^{\frac{1}{2}}(\zeta w)_{x}, \partial_{x}^{2 s} v\right)\right| & \leq C \delta^{\frac{1}{2}}\left(\|w\|_{s+1}^{2}+\|w\|_{s+1}\|\zeta\|_{s+1}\right)\left\|\partial_{x}^{s} v\right\| \\
& \leq C \delta^{\frac{1}{2}}\left(\left\|\partial_{x}^{s} v\right\|^{2}+1\right) .
\end{aligned}
$$

Combining the above estimates, we have

$$
\frac{\mathrm{d}}{\mathrm{d} t}\left\|\partial_{x}^{s} v\right\|^{2} \leq C \delta^{\frac{1}{4}}\left(1+\|h\|_{s-2}^{2}+\left\|\partial_{x}^{s} v\right\|^{4}\right) .
$$

Proceeding as in the proof of Proposition 2.4 in [37], we can choose $\delta_{0} \in(0,1)$ so small such that for any $\delta<\delta_{0}$, $s>\frac{1}{2}$

$$
\left\|\partial_{x}^{s} v(t)\right\|^{2} \leq C \delta^{\frac{1}{4}} \quad \text { for } 0<t \leq 1 \wedge\left(\delta^{-1} T\right) .
$$

According to (3.14) and (3.15), we have

$$
\|v(1)\|_{s}^{2} \leq C \delta^{\frac{1}{4}}
$$


namely,

$$
\left\|u(\delta)-\left(u_{0}+\eta-\zeta \zeta_{x}\right)\right\|_{s}^{2} \leq C \delta^{\frac{1}{4}} .
$$

The proof of Proposition 3.7 is complete.

\subsection{Proof of Theorem 3.3}

The proof of Theorem 3.3 is analogous to Theorem 3.3 in [37], the proof is divided into four steps.

Step 1. Controllability in small time to $u_{0}+\mathcal{H}_{0}$. Let us assume for the moment that $u_{0} \in H^{s+2}(\mathbb{T})$. For any $\eta \in \mathcal{H}_{0}$, applying Proposition 3.7 for the couple $(\eta, 0)$, we see that

$$
\mathcal{R}_{\delta}\left(u_{0}, 0, h+\delta^{-1} \eta\right) \rightarrow u_{0}+\eta \text { in } H^{s}(\mathbb{T}) \text { as } \delta \rightarrow 0 .
$$

It follows from the above limit that for any $\varepsilon>0$, there is a time $0<\theta_{1}<T$ such that $\hat{\eta}=\theta_{1}^{-1} \eta \in L^{2}(0, T ; \mathcal{H})$ and

$$
\left\|\mathcal{R}_{\theta_{1}}\left(u_{0}, h+\hat{\eta}\right)-u_{0}-\eta\right\|_{s}<\varepsilon
$$

Step 2. Controllability in small time to $u_{0}+\mathcal{H}_{N}$. We argue by induction. Assume that the approximate controllability of (3.1) to the set $u_{0}+\mathcal{H}_{N-1}$ is already proved. Let $\eta_{1} \in \mathcal{H}_{N}$ be of the form

$$
\eta_{1}=\eta-\sum_{m=1}^{n} \zeta_{m} \zeta_{m x}
$$

for some integer $n \geq 1$ and vectors $\eta, \zeta_{1}, \cdots, \zeta_{n} \in \mathcal{H}_{N-1}$. Applying Proposition 3.7 for the couple $\left(0, \zeta_{1}\right)$, we see that

$$
\mathcal{R}_{\delta}\left(u_{0}, \delta^{-\frac{1}{2}} \zeta_{1}, h\right) \rightarrow u_{0}-\zeta_{1} \zeta_{1 x} \text { in } H^{s}(\mathbb{T}) \text { as } \delta \rightarrow 0
$$

Using the equality

$$
\mathcal{R}_{\delta}\left(u_{0}+\delta^{-\frac{1}{2}} \zeta_{1}, 0, h\right)=\mathcal{R}_{\delta}\left(u_{0}, \delta^{-\frac{1}{2}} \zeta_{1}, h\right)+\delta^{-\frac{1}{2}} \zeta_{1}
$$

and the limit (3.17), we obtain

$$
\left\|\mathcal{R}_{\delta}\left(u_{0}+\delta^{-\frac{1}{2}} \zeta_{1}, 0, h\right)-u_{0}+\zeta_{1} \zeta_{1 x}-\delta^{-\frac{1}{2}} \zeta_{1}\right\|_{s} \rightarrow 0 \text { as } \delta \rightarrow 0 .
$$

Combining this with the fact that $\eta, \zeta_{1} \in \mathcal{H}_{N-1}$, the induction hypothesis, and Proposition 3.6, we can find a small time $\theta_{2}>0$ and a control $\hat{\eta}_{1} \in L^{2}(0, T ; \mathcal{H})$ such that

$$
\left\|\mathcal{R}_{\theta_{2}}\left(u_{0}, h+\hat{\eta}_{1}\right)-u_{0}-\eta+\zeta_{1} \zeta_{1 x}\right\|_{s}<\varepsilon
$$

Iterating this argument successively for the vectors $\zeta_{2}, \cdots, \zeta_{n}$, we construct a small time $\theta>0$ and a control $\hat{\eta}_{1} \in L^{2}(0, T ; \mathcal{H})$ satisfying

$$
\left\|\mathcal{R}_{\theta}\left(u_{0}, h+\hat{\eta}_{1}\right)-u_{0}-\eta+\zeta_{1} \zeta_{1 x}+\cdots+\zeta_{n} \zeta_{n x}\right\|_{s}=\left\|\mathcal{R}_{\theta}\left(u_{0}, h+\hat{\eta}_{1}\right)-u_{0}-\eta_{1}\right\|_{s}<\varepsilon,
$$

where we used (3.16). This proves the approximate controllability in small time to any point in $u_{0}+\mathcal{H}_{N}$. 
Step 3. Global controllability in small time. Now let $u_{1} \in H^{s}(\mathbb{T})$ be arbitrary. As $\mathcal{H}_{\infty}$ is dense in $H^{s}(\mathbb{T})$, there is an integer $N \geq 1$ and point $\hat{u}_{1} \in u_{0}+\mathcal{H}_{N}$ such that

$$
\left\|u_{1}-\hat{u}_{1}\right\|_{s}<\frac{\varepsilon}{2}
$$

By the results of Steps 1 and 2 , for any $\varepsilon>0$, there is a time $\theta>0$ and a control $\hat{\eta} \in L^{2}(0, T ; \mathcal{H})$ satisfying

$$
\left\|\mathcal{R}_{\theta}\left(u_{0}, h+\hat{\eta}\right)-\hat{u}_{1}\right\|_{s}<\frac{\varepsilon}{2} .
$$

Combining this with (3.18), we get approximate controllability in small time to $u_{1}$. Due to Proposition 3.6 and the fact that the space $H^{s+2}(\mathbb{T})$ is dense in $H^{s}(\mathbb{T})$, we can also obtain small time approximate controllability starting from $u_{0} \in H^{s}(\mathbb{T})$.

Step 4. Global controllability in fixed time T. Applying the result of Step 3, for any $\varepsilon>0$, there is a time $T_{1}>0$ and a control $\eta_{1} \in L^{2}\left(0, T_{1} ; \mathcal{H}\right)$ satisfying

$$
\left\|\mathcal{R}_{T_{1}}\left(u_{0}, h+\eta_{1}\right)-u_{1}\right\|_{s}<\frac{\varepsilon}{2}
$$

Take $v_{1}=\mathcal{R}_{T_{1}}\left(u_{0}, h+\eta_{1}\right)$. According to Proposition 3.6, we can find $\tau>0$ such that for $t \in[0, \tau]$,

$$
\left\|\mathcal{R}_{t}\left(v_{1}, h\right)-v_{1}\right\|_{s}<\frac{\varepsilon}{2}
$$

Define a control function

$$
\eta_{2}(t)= \begin{cases}\eta_{1}(t) & t \in\left[0, T_{1}\right] \\ 0 & t \in\left(T_{1}, T_{1}+\tau\right]\end{cases}
$$

then, it follows that

$$
\left\|\mathcal{R}_{T_{1}+t}\left(u_{0}, h+\eta_{2}\right)-u_{1}\right\|_{s}<\varepsilon, \quad \forall t \in[0, \tau]
$$

If $T_{1}+\tau \geq T$, then the proof is complete. Otherwise, take $v_{2}=\mathcal{R}_{T_{1}+\tau}\left(u_{0}, h+\eta_{2}\right)$, by the result of Step 3 , there is a time $T_{2}>0$ and a control $\eta_{3} \in L^{2}\left(0, T_{2} ; \mathcal{H}\right)$ satisfying

$$
\left\|\mathcal{R}_{T_{2}}\left(v_{2}, h+\eta_{3}\right)-u_{1}\right\|_{s}<\frac{\varepsilon}{2}
$$

Take $v_{3}=\mathcal{R}_{T_{2}}\left(v_{2}, h+\eta_{3}\right)$, applying again Proposition 3.6, for the same $\tau$, if $t \in[0, \tau]$, we have

$$
\left\|\mathcal{R}_{t}\left(v_{3}, h\right)-v_{3}\right\|_{s}<\frac{\varepsilon}{2}
$$

Define the control function

$$
\eta_{4}(t)= \begin{cases}\eta_{2}(t) & t \in\left[0, T_{1}+\tau\right], \\ \eta_{3}\left(t-T_{1}-\tau\right) & t \in\left(T_{1}+\tau, T_{1}+T_{2}+\tau\right], \\ 0 & t \in\left(T_{1}+T_{2}+\tau, T_{1}+T_{2}+2 \tau\right]\end{cases}
$$


we can see that

$$
\left\|\mathcal{R}_{T_{1}+T_{2}+\tau+t}\left(u_{0}, h+\eta_{4}\right)-u_{1}\right\|_{s}<\varepsilon, \quad \forall t \in[0, \tau]
$$

Again, if $T_{1}+T_{2}+2 \tau \geq T$, then the proof is complete. Otherwise, after a finite number of iterations, we complete the proof of Theorem 3.3.

\subsection{Proof of Proposition 3.4}

The proof is divided into four steps.

Step 1. We show that

$$
\sin (2 m x), \cos (2 m x) \in \mathcal{H}_{1} \quad \text { for } m \in \mathcal{I}
$$

Indeed, the case $m=0$ is obvious. If $m \neq 0$, we have

$$
\begin{aligned}
\sin (2 m x) & =2 \sin (m x) \cos (m x)=\frac{2}{m} \sin (m x)[\sin (m x)]_{x} \in \mathcal{H}_{1} \\
\cos (2 m x) & =\cos ^{2}(m x)-\sin ^{2}(m x) \\
& =[\cos (m x)-\sin (m x)][\cos (m x)+\sin (m x)] \\
& =-\frac{1}{m}[\cos (m x)-\sin (m x)][\cos (m x)-\sin (m x)]_{x} \in \mathcal{H}_{1}
\end{aligned}
$$

Step 2. We prove

$$
\cos (l+m) x \in \mathcal{H}_{1} \quad \text { for } l, m \in \mathcal{I} \text {. }
$$

Indeed, if $l+m=0, \cos (l+m) x \in \mathcal{H}_{0} \subset \mathcal{H}_{1}$.

If $l-m=0$, by the result in step 1 , we have $\cos (l+m) x \in \mathcal{H}_{1}$.

If $|l| \neq|m|$, since

$$
\cos (l+m) x=\cos (l x) \cos (m x)-\sin (l x) \sin (m x)
$$

our task is reduced to show that $\cos (l x) \cos (m x), \sin (l x) \sin (m x) \in \mathcal{H}_{1}$.

Taking

$$
\begin{aligned}
& u_{1}(x)=\sin (l x)+\cos (m x) \in \mathcal{H}_{0}, \\
& u_{2}(x)=\sin (m x)+\cos (l x) \in \mathcal{H}_{0},
\end{aligned}
$$

it follows that

$$
\begin{aligned}
u_{1}(x) u_{1 x}(x) & =[\sin (l x)+\cos (m x)][l \cos (l x)-m \sin (m x)] \\
& =l \sin (l x) \cos (l x)-m \sin m x \cos (m x)-m \sin (l x) \sin (m x)+l \cos (l x) \cos (m x) \\
& =\frac{l}{2} \sin (2 l x)-\frac{m}{2} \sin (2 m x)-m \sin (l x) \sin (m x)+l \cos (l x) \cos (m x), \\
u_{2}(x) u_{2 x}(x) & =[\sin (m x)+\cos (l x)][m \cos (m x)-l \sin (l x)] \\
& =m \sin (m x) \cos (m x)-l \sin l x \cos (l x)-l \sin (l x) \sin (m x)+m \cos (l x) \cos (m x) \\
& =\frac{m}{2} \sin (2 m x)-\frac{l}{2} \sin (2 l x)-l \sin (l x) \sin (m x)+m \cos (l x) \cos (m x) .
\end{aligned}
$$


By the definition of $\mathcal{H}_{1}$ and the results in Step 1, we can obtain that

$$
\begin{aligned}
& u_{1}(x) u_{1 x}(x)-\frac{l}{2} \sin (2 l x)+\frac{m}{2} \sin (2 m x) \in \mathcal{H}_{1}, \\
& u_{2}(x) u_{2 x}(x)-\frac{m}{2} \sin (2 m x)+\frac{l}{2} \sin (2 l x) \in \mathcal{H}_{1} .
\end{aligned}
$$

According to (3.19), we can get the expressions of $\cos (l x) \cos (m x)$ and $\sin (l x) \sin (m x)$, this means that $\cos (l x) \cos (m x), \sin (l x) \sin (m x) \in \mathcal{H}_{1}$.

Step 3. We prove

$$
\sin (l+m) x \in \mathcal{H}_{1} \quad \text { for } l, m \in \mathcal{I} .
$$

Indeed, if $l+m=0, \sin (l+m) x \in \mathcal{H}_{0} \subset \mathcal{H}_{1}$.

If $l-m=0$, by the result in step 1 , we have $\sin (l+m) x \in \mathcal{H}_{1}$.

If $|l| \neq|m|$, since

$$
\sin (l+m) x=\sin (l x) \cos (m x)+\sin (m x) \cos (l x),
$$

our task is reduced to show that $\sin (l x) \cos (m x), \sin (m x) \cos (l x) \in \mathcal{H}_{1}$.

Taking

$$
\begin{aligned}
& u_{3}(x)=\sin (l x)+\sin (m x) \in \mathcal{H}_{0}, \\
& u_{4}(x)=\cos (l x)+\cos (m x) \in \mathcal{H}_{0},
\end{aligned}
$$

proceeding as in (3.19), we have

$$
\begin{aligned}
& u_{3}(x) u_{3 x}(x)=\frac{l}{2} \sin (2 l x)+\frac{m}{2} \sin (2 m x)+m \sin (l x) \cos (m x)+l \sin (m x) \cos (l x), \\
& u_{4}(x) u_{4 x}(x)=-\frac{l}{2} \sin (2 l x)-\frac{m}{2} \sin (2 m x)-l \sin (l x) \cos (m x)-m \sin (m x) \cos (l x) .
\end{aligned}
$$

Since

$$
\begin{aligned}
& u_{3}(x) u_{3 x}(x)-\frac{l}{2} \sin (2 l x)-\frac{m}{2} \sin (2 m x) \in \mathcal{H}_{1} \\
& u_{4}(x) u_{4 x}(x)+\frac{l}{2} \sin (2 l x)+\frac{m}{2} \sin (2 m x) \in \mathcal{H}_{1}
\end{aligned}
$$

It follows from (3.20) that $\sin (l x) \cos (m x), \sin (m x) \cos (l x) \in \mathcal{H}_{1}$.

Step 4. Note that for any $m \in \mathcal{I}, \sin (-m x), \cos (-m x) \in \mathcal{H}$, thus by the results in Step 2 and Step 3, we have

$$
\cos (l \pm m) x, \sin (l \pm m) x \in \mathcal{H}_{1} \quad \text { for } l, m \in \mathcal{I} .
$$

Since $\mathcal{I}$ is a generator, repeating the above steps, we derive

$$
\operatorname{span}\{\sin (m x), \cos (m x): m \in \mathbb{Z}\} \subset \mathcal{H}_{\infty},
$$

this implies that $\mathcal{H}(\mathcal{I})$ is saturating.

The proof of Proposition 3.4 is complete. 


\subsection{Proof of Lemma 3.5}

Let $y$ be the solution to the system

$$
\begin{cases}y_{t}+y_{x x x x}=f & \text { in } \mathbb{T} \times(0, T) \\ y(0)=y_{0} & \text { in } \mathbb{T}\end{cases}
$$

Taking the scalar product in $L^{2}(\mathbb{T})$ of equation (3.21) with $y+\partial_{x}^{2 s} y$, it follows that

$$
\frac{1}{2} \frac{\mathrm{d}}{\mathrm{d} t}\left(\|y\|^{2}+\left\|\partial_{x}^{s} y\right\|^{2}\right)+\left\|y_{x x}\right\|^{2}+\left\|\partial_{x}^{s+2} y\right\|^{2}=\left(y+\partial_{x}^{2 s} y, f\right)
$$

this yields

$$
\|y(t)\|_{s}^{2}+\int_{0}^{t}\left\|\partial_{x}^{s+2} y(r)\right\|^{2} \mathrm{~d} r \leq C\left(\left\|y_{0}\right\|_{s}^{2}+\int_{0}^{t}\left(y+\partial_{x}^{2 s} y, f\right) \mathrm{d} r\right) .
$$

Since

$$
C \int_{0}^{t}\left(y+\partial_{x}^{2 s} y, f\right) \mathrm{d} r \leq C \int_{0}^{t}\left(\|y(r)\|^{2}+\|f(r)\|^{2}+\left\|\partial_{x}^{s-2} f(r)\right\|^{2}\right) \mathrm{d} r+\frac{1}{2} \int_{0}^{t}\left\|\partial_{x}^{s+2} y(r)\right\|^{2} \mathrm{~d} r
$$

we have

$$
\|y(t)\|_{s}^{2}+\int_{0}^{t}\left\|\partial_{x}^{s+2} y(r)\right\|^{2} \mathrm{~d} r \leq C\left[\left\|y_{0}\right\|_{s}^{2}+\int_{0}^{t}\left(\|y(r)\|^{2}+\|f(r)\|_{s-2}^{2}\right) \mathrm{d} r\right] .
$$

By applying Gronwall's inequality, we have

$$
\|y\|_{C\left([0, T] ; H^{s}(\mathbb{T})\right)} \leq C\left(\left\|y_{0}\right\|_{s}+\|f\|_{L^{2}\left(0, T ; H^{s-2}(\mathbb{T})\right.}\right) .
$$

Plugging this estimate into (3.22), it is shown that

$$
\|y\|_{L^{2}\left(0, T ; H^{s+2}(\mathbb{T})\right.} \leq C\left(\left\|y_{0}\right\|_{s}+\|f\|_{L^{2}\left(0, T ; H^{s-2}(\mathbb{T})\right.}\right) .
$$

Combining the above estimates, we conclude that

$$
\|y\|_{\mathcal{X}_{T, s}} \leq C\left(\left\|y_{0}\right\|_{s}+\|f\|_{L^{2}\left(0, T ; H^{s-2}(\mathbb{T})\right.}\right) .
$$

If we take $f=0$ in (3.21), the solution $y$ to (3.21) is $S(t) y_{0}$, it satisfies the first estimate in (3.3).

If we take $y_{0}=0$ in $(3.21)$, the solution $y$ to $(3.21)$ is $\int_{0}^{t} S(t-s) f(s) \mathrm{d} s$, it satisfies the second estimate in (3.3).

\section{Proof of Theorem 1.2}

In this section, we will establish the irreducibility of stochastic KSE. 


\subsection{Continuity of solving operator for deterministic KSE}

We consider the following integral equation:

$$
u(t)-u_{0}+\int_{0}^{t}\left(u_{x x x x}+u_{x x}+u u_{x}\right)(r) \mathrm{d} r=g(t) .
$$

For $s \in \mathbb{R}$, we define spaces

$$
X^{s}:=C\left([0, T] ; H^{s}(\mathbb{T})\right), \quad V_{s}:=\left\{\zeta \in X^{s} \mid \zeta(0)=0\right\}
$$

By the same argument as in Exercise 2.1.27 of [33], we know that (4.1) has a unique solution $u \in$ $C\left([0, T], L^{2}(\mathbb{T})\right) \cap L^{2}\left(0, T ; H^{2}(\mathbb{T})\right)$ if $u_{0} \in L^{2}(\mathbb{T})$ and $g \in V_{2}$.

Proposition 4.1. For any $T>0$, the solution of equation (4.1) satisfies the following estimates: (i). If $u_{0} \in L^{2}(\mathbb{T})$ and $g \in V_{2}$,

$$
\sup _{0 \leq t \leq T}\|u(t)\|^{2}+\int_{0}^{T}\|u(s)\|_{2}^{2} \mathrm{~d} s \leq C\left(T,\left\|u_{0}\right\|,\|g\|_{V_{2}}\right) .
$$

(ii). If $u_{0} \in H^{2}(\mathbb{T})$ and $g \in V_{4}$,

$$
\sup _{0 \leq t \leq T}\|u(t)\|_{2}^{2}+\int_{0}^{T}\|u(s)\|_{4}^{2} \mathrm{~d} s \leq C\left(T,\left\|u_{0}\right\|_{2},\|g\|_{V_{4}}\right) .
$$

Proof. (i). Making the substitution $u(t)=y(t)+g(t)$, then $y$ satisfies the equation

$$
\begin{cases}y_{t}+y_{x x x}+y_{x x}+(y+g)(y+g)_{x}=-g_{x x x x}-g_{x x} & \text { in } \mathbb{T} \times(0, T), \\ y(0)=u_{0} & \text { in } \mathbb{T} .\end{cases}
$$

Multiplying (4.4) with $y$, we have

$$
\frac{1}{2} \frac{\mathrm{d}}{\mathrm{d} t}\|y\|^{2}+\left\|y_{x x}\right\|^{2}=-\left(y_{x x}, y\right)-\left((y g)_{x}, y\right)-\left(g g_{x}+g_{x x x x}+g_{x x}, y\right) .
$$

The right hand side can be estimated as follows:

$$
\begin{aligned}
\left|\left(y_{x x}, y\right)\right| & \leq \frac{1}{4}\left\|y_{x x}\right\|^{2}+\|y\|^{2}, \\
\left|\left((y g)_{x}, y\right)\right| & =\left|\int_{\mathbb{T}} g y y_{x} \mathrm{~d} x\right|=\frac{1}{2}\left|\int_{\mathbb{T}} g_{x} y^{2} \mathrm{~d} x\right| \leq C\|g\|_{2}\|y\|^{2}, \\
\left|\left(g g_{x}+g_{x x x x}+g_{x x}, y\right)\right| & \leq\left|\left(g g_{x}+g_{x x}, y\right)\right|+\left|\left(g_{x x}, y_{x x}\right)\right| \\
& \leq \frac{1}{4}\left\|y_{x x}\right\|^{2}+C\|y\|^{2}+C\left(\|g\|_{1}^{4}+\|g\|_{2}^{2}\right) .
\end{aligned}
$$

Thus, we can obtain that

$$
\frac{\mathrm{d}}{\mathrm{d} t}\|y\|^{2}+\left\|y_{x x}\right\|^{2} \leq C\left(\|g\|_{2}+1\right)\|y\|^{2}+C\left(\|g\|_{1}^{4}+\|g\|_{2}^{2}\right) .
$$


It follows from Gronwall's inequality that

$$
\sup _{0 \leq t \leq T}\|y(t)\|^{2}+\int_{0}^{T}\left\|y_{x x}(s)\right\|^{2} \mathrm{~d} s \leq C\left(T,\left\|u_{0}\right\|,\|g\|_{V_{2}}\right) .
$$

Due to the fact that $u(t)=y(t)+g(t)$, we can obtain (4.2).

(ii). Multiplying (4.4) with $y_{x x x x}$, we have

$$
\begin{aligned}
& \frac{1}{2} \frac{\mathrm{d}}{\mathrm{d} t}\left\|y_{x x}\right\|^{2}+\left\|y_{x x x x}\right\|^{2} \\
= & -\left(y_{x x}, y_{x x x x}\right)-\left(y y_{x}, y_{x x x x}\right)-\left((y g)_{x}, y_{x x x x}\right)-\left(g g_{x}+g_{x x x x}+g_{x x}, y_{x x x x}\right) \\
= & I_{1}+I_{2}+I_{3}+I_{4} .
\end{aligned}
$$

According to Lemma 2.3, the following holds

$$
\begin{aligned}
\left|I_{1}\right| & \leq \varepsilon\left\|y_{x x x x}\right\|^{2}+C(\varepsilon)\left\|y_{x x}\right\|^{2}, \\
\left|I_{2}\right| & \leq\|y\|\left\|y_{x}\right\|_{L^{\infty}}\left\|y_{x x x x}\right\| \\
& \leq C\|y\|\|y\|_{2}\left\|y_{x x x x}\right\| \\
& \leq C\|y\|^{2}\left\|y_{x x x x}\right\|+C\|y\|\left\|y_{x x}\right\|\left\|y_{x x x x}\right\| \\
& \leq \varepsilon\left\|y_{x x x x}\right\|^{2}+C(\varepsilon)\left(\|y\|^{4}+\|y\|^{2}\left\|y_{x x}\right\|^{2}\right), \\
\left|I_{3}\right| & \leq C\|y\|_{1}\|g\|_{1}\left\|y_{x x x x}\right\| \\
& \leq C\|y\|\|g\|_{1}\left\|y_{x x x x}\right\|+C\left\|y_{x x}\right\|\|g\|_{1}\left\|y_{x x x x}\right\| \\
& \leq \varepsilon\left\|y_{x x x x}\right\|^{2}+C(\varepsilon)\|g\|_{1}^{2}\left(\|y\|^{2}+\left\|y_{x x}\right\|^{2}\right), \\
\left|I_{4}\right| & \leq C\left(\|g\|_{1}^{2}+\|g\|_{4}\right)\left\|y_{x x x x}\right\| \\
& \leq \varepsilon\left\|y_{x x x x}\right\|^{2}+C(\varepsilon)\left(\|g\|_{1}^{4}+\|g\|_{4}^{2}\right) .
\end{aligned}
$$

Taking $\varepsilon$ sufficiently small, it follows that

$$
\frac{\mathrm{d}}{\mathrm{d} t}\left\|y_{x x}\right\|^{2}+\left\|y_{x x x x}\right\|^{2} \leq C\left(1+\|y\|^{2}+\|g\|_{1}^{2}\right)\left\|y_{x x}\right\|^{2}+C\left(\|y\|^{4}+\|g\|_{1}^{2}\|y\|^{2}+\|g\|_{1}^{4}+\|g\|_{4}^{2}\right)
$$

Applying (4.5) and Gronwall's inequality, we can obtain that

$$
\sup _{0 \leq t \leq T}\left\|y_{x x}(t)\right\|^{2}+\int_{0}^{T}\left\|y_{x x x x}(s)\right\|^{2} \mathrm{~d} s \leq C\left(T,\left\|u_{0}\right\|_{2},\|g\|_{V_{4}}\right) .
$$

This implies (4.3).

The proof of Proposition 4.1 is complete.

Proposition 4.2. Let $u=F(g)$ be the solution of (4.1), then

(i). If $u_{0} \in L^{2}(\mathbb{T})$, the map $F: V_{2} \rightarrow X^{0}, g \mapsto u$ is continuous;

(ii). If $u_{0} \in H^{2}(\mathbb{T})$, the map $F: V_{4} \rightarrow X^{2}, g \mapsto u$ is continuous.

Proof. (i). For any fixed $R>0$, let $g_{i} \in V_{2},\left\|g_{i}\right\|_{V_{2}} \leq R(i=1,2)$ and $u_{i}$ be the solution of (4.1) with $g=g_{i}$. Define $y_{i}:=u_{i}-g_{i}(i=1,2), g:=g_{1}-g_{2}, y:=y_{1}-y_{2}$, then $y$ satisfies the following equation:

$$
\begin{cases}y_{t}+y_{x x x x}+y_{x x}+u_{1} u_{1 x}-u_{2} u_{2 x}=-g_{x x x x}-g_{x x} & \text { in } \mathbb{T} \times(0, T), \\ y(0)=0 & \text { in } \mathbb{T} .\end{cases}
$$


Multiplying (4.6) with $y$ yields

$$
\begin{aligned}
\frac{1}{2} \frac{\mathrm{d}}{\mathrm{d} t}\|y\|^{2}+\left\|y_{x x}\right\|^{2} & =-\left(y_{x x}, y\right)-\left(u_{1} u_{1 x}-u_{2} u_{2 x}, y\right)-\left(g_{x x x x}+g_{x x}, y\right) \\
& \leq \varepsilon\left\|y_{x x}\right\|^{2}+C(\varepsilon)\left(\|y\|^{2}+\|g\|_{2}^{2}\right)-\left(u_{1} u_{1 x}-u_{2} u_{2 x}, y\right) .
\end{aligned}
$$

It remains only to estimate $\left(u_{1} u_{1 x}-u_{2} u_{2 x}, y\right)$. Since

$$
\begin{aligned}
\left|\left(u_{1} u_{1 x}-u_{2} u_{2 x}, y\right)\right| & =\left|\left(u_{1}(y+g)_{x}+(y+g) u_{2 x}, y\right)\right| \\
& \leq\left|\left(u_{1} y_{x}, y\right)\right|+\left|\left(u_{1} g_{x}, y\right)\right|+\left|\left(y u_{2 x}, y\right)\right|+\left|\left(g u_{2 x}, y\right)\right|,
\end{aligned}
$$

it is shown that

$$
\begin{aligned}
&\left|\left(u_{1} y_{x}, y\right)\right|+\left|\left(y u_{2 x}, y\right)\right| \leq C \int_{\mathbb{T}}\left(\left|u_{1}\right|+\left|u_{2}\right|\right)\left|y \| y_{x}\right| \mathrm{d} x \\
& \leq C\left(\left\|u_{1}\right\|+\left\|u_{2}\right\|\right)\|y\|\left\|y_{x}\right\|_{L^{\infty}(\mathbb{T})} \\
& \leq C\left(\left\|u_{1}\right\|+\left\|u_{2}\right\|\right)\|y\|\|y\|_{2} \\
& \leq C\left(\left\|u_{1}\right\|+\left\|u_{2}\right\|\right)\|y\|^{2}+C\left(\left\|u_{1}\right\|+\left\|u_{2}\right\|\right)\|y\|\left\|y_{x x}\right\| \\
& \leq \varepsilon\left\|y_{x x}\right\|^{2}+C(\varepsilon)\left(1+\left\|u_{1}\right\|^{2}+\left\|u_{2}\right\|^{2}\right)\|y\|^{2} \\
&\left|\left(u_{1} g_{x}, y\right)\right|+\left|\left(g u_{2 x}, y\right)\right| \leq C \int_{\mathbb{T}}\left(\left|u_{1}\right|+\left|u_{2}\right|\right)\left|g_{x} \| y\right| \mathrm{d} x+\int_{\mathbb{T}}\left|g u_{2} y_{x}\right| \mathrm{d} x \\
& \leq C\left(\left\|u_{1}\right\|+\left\|u_{2}\right\|\right)\|g\|_{2}\|y\|+C\|g\|_{1}\left\|u_{2}\right\|\left\|y_{x}\right\| \\
& \leq C\left(\left\|u_{1}\right\|+\left\|u_{2}\right\|\right)\|g\|_{2}\|y\|+C\|g\|_{1}\left\|u_{2}\right\|\left(\|y\|+\left\|y_{x x}\right\|\right) \\
& \leq C\left(\left\|u_{1}\right\|+\left\|u_{2}\right\|\right)\|g\|_{2}\|y\|+C\|g\|_{1}\left\|u_{2}\right\|\left\|y_{x x}\right\| \\
& \leq \varepsilon\left\|y_{x x}\right\|^{2}+C\|y\|^{2}+C(\varepsilon)\left(\left\|u_{1}\right\|^{2}+\left\|u_{2}\right\|^{2}\right)\|g\|_{2}^{2} .
\end{aligned}
$$

Combining the above estimates and choosing $0<\varepsilon \ll 1$, we get

$$
\begin{aligned}
\frac{\mathrm{d}}{\mathrm{d} t}\|y\|^{2}+\left\|y_{x x}\right\|^{2} & \leq C\left(1+\left\|u_{1}\right\|^{2}+\left\|u_{2}\right\|^{2}\right)\|y\|^{2}+C\left(1+\left\|u_{1}\right\|^{2}+\left\|u_{2}\right\|^{2}\right)\|g\|_{2}^{2} \\
& \leq C\left(T,\left\|u_{0}\right\|, R\right)\|y\|^{2}+C\left(T,\left\|u_{0}\right\|, R\right)\|g\|_{2}^{2},
\end{aligned}
$$

here we have used (4.2). It follows from Gronwall's inequality that

$$
\|y\|_{X^{0}}^{2} \leq C\left(T,\left\|u_{0}\right\|, R\right)\|g\|_{V_{2}}^{2}
$$

which leads to

$$
\left\|u_{1}-u_{2}\right\|_{X^{0}} \leq C\left(T,\left\|u_{0}\right\|, R\right)\left\|g_{1}-g_{2}\right\|_{V_{2}} .
$$

(ii). For any fixed $R>0$, let $g_{i} \in V_{4},\left\|g_{i}\right\|_{V_{4}} \leq R(i=1,2)$.

Multiplying (4.6) with $y_{x x x x}$, we have

$$
\begin{aligned}
\frac{1}{2} \frac{\mathrm{d}}{\mathrm{d} t}\left\|y_{x x}\right\|^{2}+\left\|y_{x x x x}\right\|^{2} & =-\left(y_{x x}, y_{x x x x}\right)-\left(u_{1} u_{1 x}-u_{2} u_{2 x}, y_{x x x x}\right)-\left(g_{x x x x}+g_{x x}, y_{x x x x}\right) \\
& \leq \varepsilon\left\|y_{x x x x}\right\|^{2}+C(\varepsilon)\left(\left\|y_{x x}\right\|^{2}+\|g\|_{4}^{2}\right)-\left(u_{1} u_{1 x}-u_{2} u_{2 x}, y_{x x x x}\right) .
\end{aligned}
$$


It is sufficient to estimate $\left(u_{1} u_{1 x}-u_{2} u_{2 x}, y_{x x x x}\right)$. Proceeding as in the proof (i), we can obtain that

$$
\begin{aligned}
& \left|\left(u_{1} u_{1 x}-u_{2} u_{2 x}, y_{x x x x}\right)\right| \\
\leq & \left|\left(u_{1} y_{x}, y_{x x x x}\right)\right|+\left|\left(u_{1} g_{x}, y_{x x x x}\right)\right|+\left|\left(y u_{2 x}, y_{x x x x}\right)\right|+\left|\left(g u_{2 x}, y_{x x x x}\right)\right| \\
\leq & \left(\left\|u_{1}\right\|_{L^{\infty}(\mathbb{T})}\left\|y_{x}\right\|+\left\|u_{1}\right\|_{L^{\infty}(\mathbb{T})}\left\|g_{x}\right\|+\|y\|_{L^{\infty}(\mathbb{T})}\left\|u_{2 x}\right\|+\|g\|_{L^{\infty}(\mathbb{T})}\left\|u_{2 x}\right\|\right)\left\|y_{x x x x}\right\| \\
\leq & C\left[\left(\left\|u_{1}\right\|_{1}+\left\|u_{2}\right\|_{1}\right)\|y\|_{1}+\left(\left\|u_{1}\right\|_{1}+\left\|u_{2}\right\|_{1}\right)\|g\|_{1}\right]\left\|y_{x x x x}\right\| \\
\leq & \varepsilon\left\|y_{x x x x}\right\|^{2}+C(\varepsilon)\left[\left(\left\|u_{1}\right\|_{1}^{2}+\left\|u_{2}\right\|_{1}^{2}\right)\|y\|_{1}^{2}+\left(\left\|u_{1}\right\|_{1}^{2}+\left\|u_{2}\right\|_{1}^{2}\right)\|g\|_{1}^{2}\right] \\
\leq & \varepsilon\left\|y_{x x x x}\right\|^{2}+C(\varepsilon)\left[\left(\left\|u_{1}\right\|_{1}^{2}+\left\|u_{2}\right\|_{1}^{2}\right)\left(\|y\|^{2}+\left\|y_{x x}\right\|^{2}\right)+\left(\left\|u_{1}\right\|_{1}^{2}+\left\|u_{2}\right\|_{1}^{2}\right)\|g\|_{1}^{2}\right] .
\end{aligned}
$$

Taking $\varepsilon$ small enough, we arrive at

$$
\frac{\mathrm{d}}{\mathrm{d} t}\left\|y_{x x}\right\|^{2}+\left\|y_{x x x x}\right\|^{2} \leq C\left(1+\left\|u_{1}\right\|_{1}^{2}+\left\|u_{2}\right\|_{1}^{2}\right)\left\|y_{x x}\right\|^{2}+C\left(1+\left\|u_{1}\right\|_{1}^{2}+\left\|u_{2}\right\|_{1}^{2}\right)\left(\|g\|_{4}^{2}+\|y\|^{2}\right) .
$$

According to the results in (i), we have

$$
\|y(t)\|^{2} \leq C\left(T,\left\|u_{0}\right\|, R\right)\|g\|_{V_{2}}^{2} \leq C\left(T,\left\|u_{0}\right\|, R\right)\|g\|_{V_{4}}^{2} .
$$

Therefore, it follows that

$$
\frac{\mathrm{d}}{\mathrm{d} t}\left\|y_{x x}\right\|^{2}+\left\|y_{x x x x}\right\|^{2} \leq C\left(T,\left\|u_{0}\right\|_{1}, R\right)\left\|y_{x x}\right\|^{2}+C\left(T,\left\|u_{0}\right\|_{1}, R\right)\|g\|_{V_{4}}^{2} .
$$

Applying Gronwall's inequality, we get

$$
\|y\|_{X^{2}}^{2} \leq C\left(T,\left\|u_{0}\right\|_{2}, R\right)\|g\|_{V_{4}}^{2}
$$

this implies

$$
\left\|u_{1}-u_{2}\right\|_{X^{2}} \leq C\left(T,\left\|u_{0}\right\|_{2}, R\right)\left\|g_{1}-g_{2}\right\|_{V_{4}} .
$$

The proof of Proposition 4.2 is complete.

\subsection{Proof of Theorem 1.2}

Denote $H:=L^{2}(\mathbb{T}), V:=H^{2}(\mathbb{T}), \mathcal{E}:=\operatorname{span}\{\sin x, \cos x, 1\}$. Set $E:=C([0, T] ; \mathcal{E})$. We know that the norms $\|\cdot\|_{E}$ and $\|\cdot\|_{V_{2}}$ are equivalent for the functions in $E$. We define $\xi(t):=b_{1} \beta_{1}(t) \sin x+b_{2} \beta_{2}(t) \cos x+b_{3} \beta_{3}(t)$.

For any $u_{0} \in H, t>0$, we have $u^{u_{0}}(t) \in V$ a.s., where $u^{u_{0}}$ is the solution to (1.1) with initial date $u_{0}$. Since $u^{u_{0}}$ is Markov in $H$, for any $u_{1} \in H, T>0, \varepsilon>0$,

$$
\begin{aligned}
\mathbb{P}\left(\left\|u^{u_{0}}(T)-u_{1}\right\|<\varepsilon\right) & =\int_{V} \mathbb{P}\left(\left\|u^{u_{0}}(T)-u_{1}\right\|<\varepsilon \mid u^{u_{0}}(t)=v\right) \mathbb{P}\left(u^{u_{0}}(t) \in \mathrm{d} v\right) \\
& =\int_{V} \mathbb{P}\left(\left\|u^{v}(T-t)-u_{1}\right\|<\varepsilon\right) \mathbb{P}\left(u^{u_{0}}(t) \in \mathrm{d} v\right)
\end{aligned}
$$

To prove that $\mathbb{P}\left(\left\|u^{u_{0}}(T)-u_{1}\right\|<\varepsilon\right)>0$, it is sufficient to prove that for any $T>0, u_{0} \in V$,

$$
\mathbb{P}\left(\left\|u^{u_{0}}(T)-u_{1}\right\|<\varepsilon\right)>0
$$


Now, we prove that (4.7) holds for any $T>0, u_{0} \in V$. We denote the solution to (4.1) with $u_{0}$ and $g$ by $u^{u_{0}}(\cdot, g)$, by this notation, the solution of (1.1) can be written as $u^{u_{0}}(\cdot, \xi)$. Let $u_{0} \in V$ and $u_{1} \in H$, since $V$ is dense in $H$, there exists a function $\tilde{u}_{1} \in V$ such that $\left\|\tilde{u}_{1}-u_{1}\right\|<\frac{\varepsilon}{4}$. According to Theorem 3.3, (3.1) is approximately controllable by $\mathcal{E}$-valued control, for any $\varepsilon>0$, there exists a control $\eta \in L^{2}(0, T ; \mathcal{E})$ such that

$$
\left\|u^{u_{0}}\left(T, \xi_{1}\right)-\tilde{u}_{1}\right\|_{2}<\frac{\varepsilon}{4}
$$

where $\xi_{1}(t):=\int_{0}^{t} \eta(s) \mathrm{d} s$, this leads to the fact

$$
\left\|u^{u_{0}}\left(T, \xi_{1}\right)-u_{1}\right\|<\frac{\varepsilon}{2}
$$

This implies that

$$
\mathbb{P}\left(\left\|u^{u_{0}}(T, \xi)-u_{1}\right\|<\varepsilon\right) \geq \mathbb{P}\left(\left\|u^{u_{0}}(T, \xi)-u^{u_{0}}\left(T, \xi_{1}\right)\right\|<\frac{\varepsilon}{2}\right) .
$$

It follows from Proposition 4.2 that there exists a positive constant $\delta>0$ such that when $\left\|\xi-\xi_{1}\right\|_{E}<\delta$,

$$
\left\|u^{u_{0}}(T, \xi)-u^{u_{0}}\left(T, \xi_{1}\right)\right\|<\frac{\varepsilon}{2}
$$

thus, we can obtain that

$$
\mathbb{P}\left(\left\|u^{u_{0}}(T, \xi)-u_{1}\right\|<\varepsilon\right) \geq \mathbb{P}\left(\left\|\xi-\xi_{1}\right\|_{E}<\delta\right) .
$$

Since the support of $\mathcal{L}(\xi)$ is $E$, we have

$$
\mathbb{P}\left(\left\|\xi-\xi_{1}\right\|_{E}<\delta\right)>0
$$

then,

$$
\mathbb{P}\left(\left\|u^{u_{0}}(T, \xi)-u_{1}\right\|<\varepsilon\right)>0
$$

The proof of Theorem 1.2 is complete.

Acknowledgements. Peng Gao would like to thank the referees and the editors for their careful comments and useful suggestions. Peng Gao would like to thank the financial support of the China Scholarship Council (No. 201806625036) and the hospitality of CNRS and IMJ, Université Paris Diderot-Paris 7 during his visit from December 2018 to November 2019. Peng Gao is supported by the Fundamental Research Funds for the Central Universities (2412020FZ022).

\section{REFERENCES}

[1] R.A. Adams and J.J.F. Fournier, Sobolev spaces. Elsevier (2003).

[2] A. Armaou and P.D. Christofides, Feedback control of the Kuramoto-Sivashinsky equation. Physica D 137 (2000) $49-61$.

[3] A.A. Agrachev and A.V. Sarychev, Navier-Stokes equations: controllability by means of low modes forcing. J. Math. Fluid Mech. 7 (2005) 108-152.

[4] A.A. Agrachev and A.V. Sarychev, Controllability of 2D Euler and Navier-Stokes equations by degenerate forcing. Commun. Math. Phys. 265 (2006) 673-697.

[5] A. Agrachev and A. Sarychev, Solid controllability in fluid dynamics. In Instability in Models Connected with Fluid Flows. I, Int. Math. Ser. (N.Y.). Springer, New York (2008) 1-35.

[6] V. Barbu, The irreducibility of transition semigroups and approximate controllability. Stochastic Partial Differential Equations and Applications-VII (2005) 21. 
[7] V. Barbu and G. Da Prato, Irreducibility of the transition semigroup associated with the two phase Stefan problem. Variational Analysis and Applications. Springer, Boston, MA (2005) 147-159.

[8] V. Barbu and G. Da Prato, Irreducibility of the transition semigroup associated with the stochastic obstacle problem. Infin. Dimens. Anal. Quantum Probab. Relat. Top. 8 (2005) 397-406.

[9] L. Bo and Y. Jiang, Large deviation for the nonlocal Kuramoto-Sivashinsky SPDE. Nonlinear Analysis: Theory Methods Appl. 82 (2013) 100-114.

[10] L. Bo, K. Shi and Y. Wang, On a nonlocal stochastic Kuramoto-Sivashinsky equation with jumps. Stochastics Dyn. 7 (2007) 439-457.

[11] P.M. Boulvard, P. Gao and V. Nersesyan, Controllability and ergodicity of 3D primitive equations driven by a finite-dimensional force (2020).

[12] N. Carreno and M.C. Santos, Stackelberg-Nash exact controllability for the Kuramoto-Sivashinsky equation. J. Differ. Equ. 266 (2019) 6068-6108.

[13] C.M. Cazacu, L.I. Ignat and A.F. Pazoto, Null-controllability of the linear Kuramoto-Sivashinsky equation on star-shaped trees. SIAM J. Control Optim. 56 (2018) 2921-2958.

[14] E. Cerpa and A. Mercado, Local exact controllability to the trajectories of the 1-D Kuramoto-Sivashinsky equation. J. Differ. Equ. 250 (2011) 2024-2044.

[15] E. Cerpa, P. Guzmán and A. Mercado, On the control of the linear Kuramoto-Sivashinsky equation. ESAIM: COCV 23 (2017) 165-194.

[16] P. Collet, J.P. Eckmann, H. Epstein et al., A global attracting set for the Kuramoto-Sivashinsky equation. Commun. Math. Phys. 152 (1993) 203-214.

[17] G. Da Prato and J. Zabczyk, Stochastic equations in infinite dimensions. Cambridge University Press (2014).

[18] Z. Dong, F.Y. Wang and L. Xu, Irreducibility and asymptotics of stochastic Burgers equation driven by $\alpha-$ stable processes. Potential Anal. 52 (2020) 371-392.

[19] J. Duan and V.J. Ervin, On the stochastic Kuramoto-Sivashinsky equation. Nonlinear Analysis: Theory Methods Appl. 44 (2001) 205-216.

[20] J. Duan and V.J. Ervin, Dynamics of a nonlocal Kuramoto-Sivashinsky equation. J. Differ. Equ. 143 (1998) $243-266$.

[21] S. Dubljevic, Boundary model predictive control of Kuramoto-Sivashinsky equation with input and state constraints. Comput. Chem. Eng. 34 (2010) 1655-1661.

[22] B. Ferrario, Invariant measures for a stochastic Kuramoto-Sivashinsky equation. Stoch. Anal. Appl. 26 (2008) $379-407$.

[23] F. Flandoli, Irreducibility of the 3-D stochastic Navier-Stokes equation. J. Funct. Anal. 149 (1997) $160-177$.

[24] F. Flandoli and B. Maslowski, Ergodicity of the 2-D Navier-Stokes equation under random perturbations. Commun. Math. Phys. 172 (1995) 119-141.

[25] P. Gao, Null controllability with constraints on the state for the 1-D Kuramoto-Sivashinsky equation. Evol. Equ. Control Theory 4 (2015) 281-296.

[26] P. Gao, Global Carleman estimates for the linear stochastic Kuramoto-Sivashinsky equations and their applications. J. Math. Anal. Appl. 464 (2018) 725-748.

[27] P. Gao, Null controllability with constraints on the state for the linear stochastic Kuramoto-Sivashinsky equation. Physica A (2020).

[28] P. Gao, Global exact controllability to the trajectories of the Kuramoto-Sivashinsky equation. Evol. Equ. Control Theory 9 (2020) 181-191.

[29] P. Gao, A new global Carleman estimate for the one-dimensional Kuramoto-Sivashinsky equation and applications to exact controllability to the trajectories and an inverse problem. Nonlinear Anal. Theory Methods Appl. (2015) $133-147$.

[30] P. Gao, Optimal distributed control of the Kuramoto-Sivashinsky equation with pointwise state and mixed control-state constraints. IMA J. Math. Control Inf. 33 (2016) 791-811.

[31] P. Gao, Averaging principle for stochastic Kuramoto-Sivashinsky equation with a fast oscillation. Discr. Continu. Dyn. Syst. 38 (2018) 5649.

[32] J. Goodman, Stability of the kuramoto-sivashinsky and related systems. Commun. Pure Appl. Math. 47 (1994) $293-306$.

[33] S. Kuksin and A. Shirikyan, Mathematics of two-dimensional turbulence. Cambridge University Press (2012).

[34] Y. Kuramoto, Diffusion-induced chaos in reaction systems. Suppl. Prog. Theor. Phys. 64 (1978) 346-367.

[35] Y. Kuramoto and T. Tsuzuki, On the formation of dissipative structures in reaction-diffusion systems. Theor. Phys. 54 (1975) 687-699.

[36] Y. Kuramoto and T. Tsuzuki, Persistent propagation of concentration waves in dissipative media far from thermal equilibrium. Prog. Theor. Phys. 55 (1976) 356-369.

[37] V. Nersesyan, Approximate controllability of nonlinear parabolic PDEs in arbitrary space dimension. Math. Control Related Fields 11 (2021) 237.

[38] V. Nersesyan, Approximate controllability of Lagrangian trajectories of the 3D Navier-Stokes system by a finite-dimensional force. Nonlinearity 28 (2015) 825-848.

[39] B. Nicolaenko, B. Scheurer and R. Temam, Some global dynamical properties of a class of pattern formation equations. Commun. Partial Differ. Equ. 14 (1989) 245-297.

[40] A. Sarychev, Controllability of the cubic Schroedinger equation via a low-dimensional source term. Math. Control Related Fields 4 (2014) 261. 
[41] G.I. Sivashinsky, Nonlinear analysis of hydrodynamic instability in laminar flames-I. Derivation of basic equations. Acta Astronaut. 4 (1977) 1177-1206.

[42] R. Temam and X.M. Wang, Estimates on the lowest dimension of inertial manifolds for the Kuramoto-Sivashinsky equation in the general case. Differ. Integr. Equ. 7 (1994) 1095-1108.

[43] R. Wang, J. Xiong and L. Xu, Irreducibility of stochastic real Ginzburg-Landau equation driven by $\alpha$-stable noises and applications. Bernoulli 23 (2017) 1179-1201.

[44] K. Yamazaki, Irreducibility of the three, and two and a half dimensional Hall-magnetohydrodynamics system. Physica D (2020).

[45] D. Yang, Dynamics for the stochastic nonlocal Kuramoto-Sivashinsky equation. J. Math. Anal. Appl. 330 (2007) 550-570.

[46] D. Yang, Random attractors for the stochastic Kuramoto-Sivashinsky equation. Stoch. Anal. Appl. 24 (2006) 1285-1303.

\section{Subscribe to Open (S2O) A fair and sustainable open access model}

This journal is currently published in open access under a Subscribe-to-Open model ( $\mathrm{S} 2 \mathrm{O}$ ). S2O is a transformative model that aims to move subscription journals to open access. Open access is the free, immediate, online availability of research articles combined with the rights to use these articles fully in the digital environment. We are thankful to our subscribers and sponsors for making it possible to publish this journal in open access, free of charge for authors.

\section{Please help to maintain this journal in open access!}

Check that your library subscribes to the journal, or make a personal donation to the S2O programme, by contacting subscribers@edpsciences.org

More information, including a list of sponsors and a financial transparency report, available at: https://www.edpsciences.org/en/maths-s2o-programme 